\title{
Measurement and Analysis of Extra Propagation Loss of Tunnel Curve
}

Bo Ai,

Ke Guan, Lei Zhang, Cesar Briso-Rodríguez, and Ruisi He

\begin{abstract}
Wave propagation experiences extra loss in curved tunnels, which is highly desired for network planning. Extensive narrow-band propagation measurements are made in two types of Madrid subway tunnels (different cross sections and curvatures) with various configurations (different frequencies and polarizations). A ray tracer validated by the straight and curved parts of the measuring tunnels is employed to simulate the reference received signal power by assuming the curved tunnel to be straight. By subtracting the measured received power in the curved tunnels from the simulated reference power, the extra loss resulting from the tunnel curve is extracted. Finally, this paper presents the figures and tables quantitatively reflecting the correlations between the extra loss and radius of curvature, frequency, polarization, and cross section, respectively. The results are valuable for statistical modeling and the involvement of the extra loss in the design and network planning of communication systems in subway tunnels.
\end{abstract}

Index Terms-Curve, propagation measurement, railway communications, subway, tunnel.

\section{INTRODUCTION}

$\mathbf{N}$ OWADAYS, a large number of public communication systems, including Global System for Mobile Communications, third-generation, Wi-Fi, World Interoperability for Microwave Access, and Long-Term Evolution (LTE), are expected to support continuous service in subway tunnels to guarantee user experience [1]. For dedicated communication systems, such as Terrestrial Trunked Radio [2], [3], Global System for Mobile Communications for Railway (GSM-R) [4], communication-based train control system [5], LTE for Railway [6], etc., high reliability, availability, maintainability, and safety [7] must to provide safety-critical data transmissions for train control in subway tunnels [8]. In the near future, various high-data-rate applications, such as real-time high-definition video surveillance, will be required to enable face recognition and emotion analysis [9]-[11] for security concerns (e.g., terrorist attacks, riots, etc). For public wireless communication systems or signaling and train control communication systems, a full understanding of the propagation is mandatory for their deployments in subway tunnels.

Over the past few decades, many works related to the propagation in tunnels have been published and have attracted intensive attention [12]-[14]. As the representatives of deterministic models, modal analysis based on waveguide theory [15], [16], models based on geometrical optics (GO) approach [17]-[19], and models based on numerical methods for solving Maxwell equations for tunnel environment, e.g., vector parabolic equation techniques [20], support an accurate way to predict the propagation characteristics in tunnels. On the other hand, empirical models, such as two-slope models [21], [22], three-slope model [23], [24], four-slope model [25], [26], and five-zone models [27], [28], etc., predict the propagation in tunnels in a fast and effective way. However, most of these models are sophisticated for the straight tunnels.

To get the insight into the influence of the tunnel curve, a few scholars conducted the corresponding research. First, for the GO approach, based on a ray launching technique, a hybrid method was presented in [29] to model the radio wave propagation in curved tunnels with a rectangular cross section. This study highlighted that the higher modes vanish quickly in the curved part. In [30], a ray optical approach based on stochastic ray launching technique was presented to model the wave propagation in curved tunnels with rectangular or arched cross sections. In [31], radio propagation in curved tunnels of rectangular or arched cross-sections was modeled. The influence of base station and tunnel parameters on the propagation slope in curved tunnels was determined qualitatively. Based on the simple image theory, a narrow-band and a wideband analysis of the propagation at 2.5 and $10 \mathrm{GHz}$ in curved tunnels were presented in [32]. In [33], an image-theory-based ray-tracing method validated by the measurements performed in a hallway of an office building was used to study ultrawideband propagation in curved tunnels. In [34], a ray-tube tracing method was presented to simulate waves propagating in curved road tunnels from base stations for mobile communications. Second, for modal analysis, in [35], it was shown that increased propagation loss is almost linearly proportional to frequency and inversely proportional to the radius of curvature. Third, for the numerical methods, in [36], the use of the parabolic equation (PE) along 
with the alternate direction implicit method in predicting the loss for curved tunnels was discussed. It was found that one of the important characteristics of the curved tunnel is the accumulation of the field near the concave wall. In [37], the radio wave propagation in curved tunnels having rectangular cross section was evaluated by using a high-frequency field prediction model. Finally, for hybrid methods, in [38], simulations of the attenuation in a tunnel as a function of the radius of curvature were made based on both waveguide methods and the GO approach. In [39], the case of a curved tunnel was studied using the Airy function representation of the modal fields.

The existing studies have already obtained some conclusions for the influence of the tunnel curve. However, it has been rare to correlate the extra loss of tunnel curve to various factors in different subway tunnel geometries and system configurations. The main contribution of this paper is to present the first-hand measurement results in the subway tunnels to quantitatively reflect the correlations between the extra loss and radius of curvature, frequency, polarization, and cross section, respectively. The extra loss extracted from the measurements are valuable for statistical modeling and can be directly added to the existing models for propagation in straight tunnels to fulfill the network planning in the curved subway tunnels (with the similar sizes, cross sections, and curvatures of the measured tunnels in this paper). Moreover, the measurements can be used to validate ray-tracing simulators that are able to predict the propagation in various curved tunnels in the future.

The remainder of this paper is organized as follows. The measurement campaign in two types of subway tunnels in Madrid is introduced in Section II. The measurement results and the method of extracting the extra loss of tunnel curve are presented in Section III. Section IV gives the analysis of the correlations between the extra loss and radius of curvature, frequency, polarization, and cross section, respectively. Discussions on the measurements and analysis are made in Section V. In Section VI, conclusions are drawn.

\section{Measurement CAMPAign}

Extensive narrow-band propagation measurements are carried out in two typical types of arched curved tunnels of Madrid's subway. In the information provided by Madrid Metro, a whole subway line is separated by many segments (marked by kilometer post) with the lengths from several decameters to several hundred meters. Each segment is with a corresponding radius of curvature that is approximated to be an integer but sufficiently accurate for engineering design. Thus, we use the radii of curvature provided by Madrid Metro, although they are the approximated values, and the curves are not perfect circular arches.

\section{A. Test System}

Fig. 1(a) provides a simplified view of the test system. The transmission system is comprised of a continuous-wave (CW) transmitter and a panel antenna. The receiving system is composed of a broadband antenna, an amplifier, and a spectrum analyzer. The analyzer sends the captured received signal power to a laptop, which is stored for later processing.

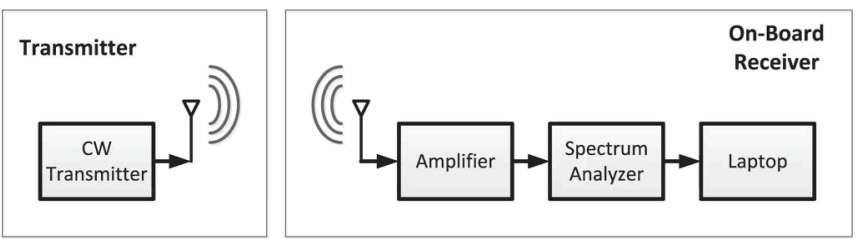

(a)

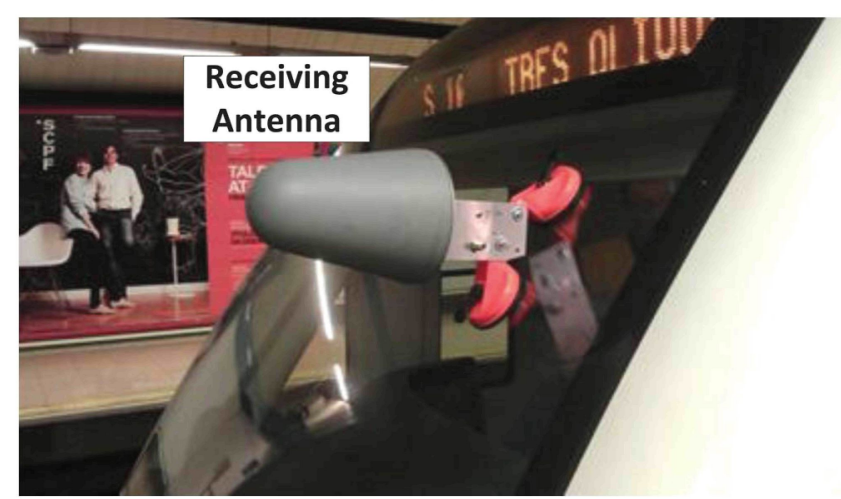

(b)

Fig. 1. (a) Block diagram of the test system. (b) Receiving antenna on the cabin of the train.

TABLE I

TEST SYSTEM

\begin{tabular}{|c|c|c|c|c|}
\hline Antenna & Model & $\begin{array}{c}\text { Frequency band } \\
(\mathrm{MHz})\end{array}$ & $\begin{array}{l}\text { Gain } \\
(\mathrm{dBi})\end{array}$ & $\begin{array}{c}\text { HPBW }\left(^{\circ}\right) \\
(\mathrm{H} / \mathrm{E})\end{array}$ \\
\hline Tx (Panel) & L-COM HG908 & $902-928$ & 8 & $75 / 65$ \\
\hline Tx (Panel) & L-COM HG2414P & $2400-2500$ & 14 & $30 / 30$ \\
\hline Tx (Panel) & L-COM HG5419P & $5470-5850$ & 19 & $16 / 16$ \\
\hline RX (Log-Periodic WB) & R\&S HL050 & $850-26500$ & 8.5 & $85 / 70$ \\
\hline Transmitter & \multicolumn{2}{|c|}{ Frequency (MHz) } & \multicolumn{2}{|c|}{ Power output $(\mathrm{mW})$} \\
\hline $\mathrm{Tx}(\mathrm{CW})$ & \multicolumn{2}{|c|}{920} & \multicolumn{2}{|c|}{$500(27 \mathrm{dBm})$} \\
\hline $\mathrm{Tx}(\mathrm{CW})$ & \multicolumn{2}{|c|}{2400} & \multicolumn{2}{|c|}{$250(24 \mathrm{dBm})$} \\
\hline Tx $(\mathrm{CW})$ & \multicolumn{2}{|c|}{5705} & \multicolumn{2}{|c|}{$160(22 \mathrm{dBm})$} \\
\hline Amplifier & \multicolumn{2}{|c|}{ Model } & \multicolumn{2}{|c|}{ Frequency band (MHz) } \\
\hline Rx Amplifier & \multicolumn{2}{|c|}{ Celwritek csa-936327 (teledyne) } & \multicolumn{2}{|c|}{$500-6000$} \\
\hline Receiver & \multicolumn{2}{|c|}{ Model } & \multicolumn{2}{|c|}{ Frequency band (MHz) } \\
\hline Spectrum analyzer & \multicolumn{2}{|c|}{ Agilent N9912A } & \multicolumn{2}{|c|}{$0.1-6000$} \\
\hline
\end{tabular}

The transmission system, located on land, is subject to a mast in the opposite way from the train to avoid possible damage. The receiving system is located on the train, with the antenna attached to the front thereof. Fig. 1(b) shows the position of the receiving antenna.

Detailed information of the test system is given by Table I, where HPBW is the abbreviation for half-power beamwidth. The transmitting antennas in this paper are directional antennas (with gains range from 8 to $19 \mathrm{~dB}$, depending of frequency), which are similar to the antennas used in the GSM-R system and other cellular communication systems (e.g., GSM) at the similar positions of real engineering-being several meters high, beside the track and near the wall inside tunnels. The receiving antenna is also similar but with a higher gain. Designing the antenna configuration like this is to obtain the results close to the engineering application. It is worth noting that the channel is a single-input-single-output (SISO) channel with directional antennas; therefore, the absolute value of received power will differ even in the same propagation environment when using different directional antennas or multiple-input-multipleoutput (MIMO) antenna arrays. However, the research topicextra loss-defined by the difference between the propagation 


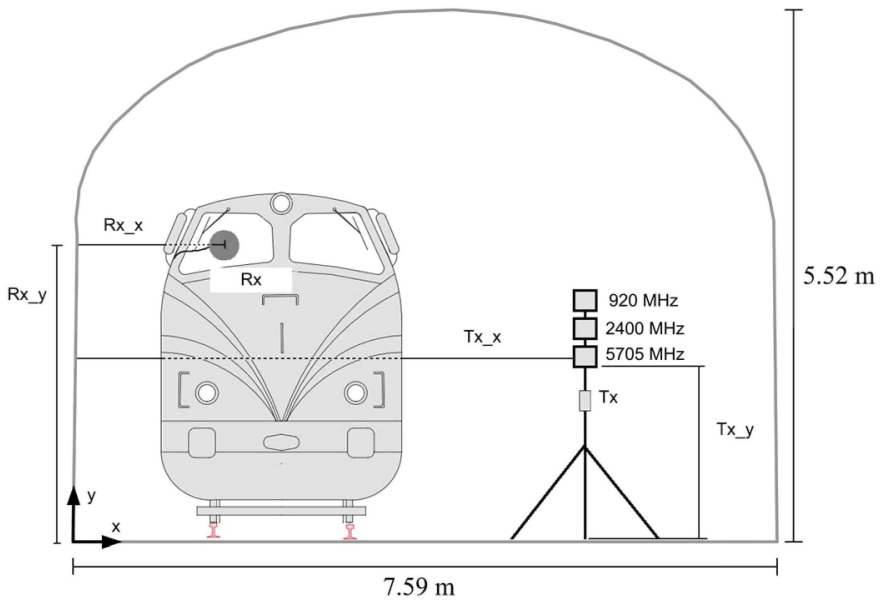

Fig. 2. Positions of Tx and Rx in the arched "Type I" tunnel.

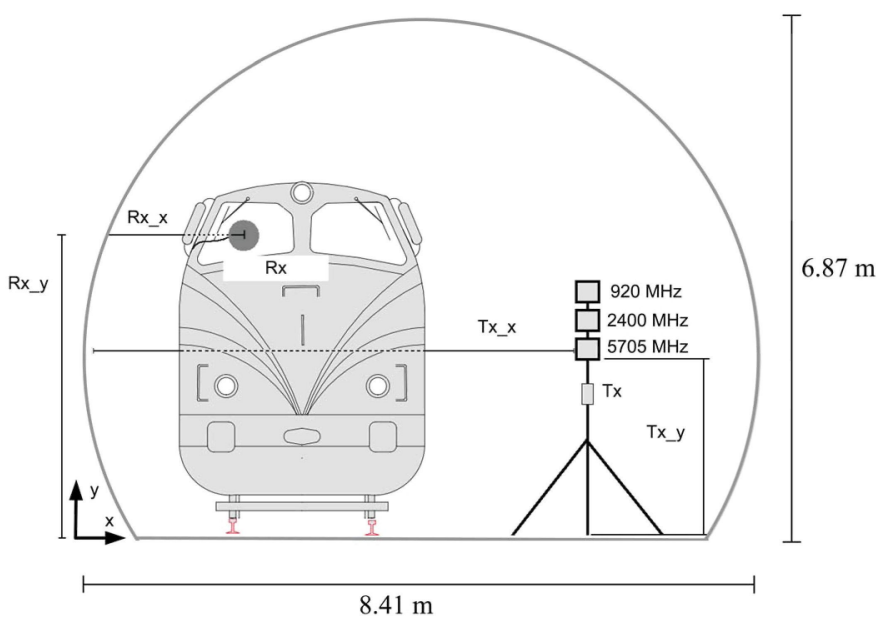

Fig. 3. Positions of Tx and Rx in the arched "Type II" tunnel.

losses in the curved and straight tunnels is isolated from the influence of antennas because, in this paper, the same patterns of antennas are embedded both in the curved tunnel cases (measurements) and straight tunnel cases (simulations).

\section{B. Measurement Environment}

The measurements are carried out in two types of arched curved tunnels of Madrid's subway. As shown in Figs. 2 and 3, there are two types of arched tunnels in the modern subway systems. To keep coherence to our previous publications, such as [40] and [41], these two types of tunnels are still named Arched "Type I" tunnel and Arched "Type II" tunnel, respectively. "Type I" consists of three plane walls and an arched roof, and "Type II" includes arched walls and roof, but a plane floor, which is more like a semi-circle. The arched "Type I" tunnel is more common in the subway tunnels built in the 1980s and 1990s, whereas the arched "Type II" tunnel is more usual in the newly built subway lines because of the widespread use of the shield machines in the constructions of modern tunnels. Relative position and configuration of the transmission system and receiving system along the train route inside two types of arched tunnels with dimensions of $7.59 \mathrm{~m} \times 5.52 \mathrm{~m}$ ("Type I" tunnel) and $8.41 \mathrm{~m} \times 6.87 \mathrm{~m}$ ("Type II") are shown in Figs. 2 and 3 as well. Positions of Tx and Rx in these two types of tunnels are given in Table II.
TABLE II

Positions of Tx AND Rx in the Measurements

\begin{tabular}{c|cc|cc}
\hline Tunnel & $\begin{array}{c}T x \_x \\
(\mathrm{~m})\end{array}$ & $\begin{array}{c}T x \_y \\
(\mathrm{~m})\end{array}$ & $\begin{array}{c}R x \_x \\
(\mathrm{~m})\end{array}$ & $\begin{array}{c}R x \_y \\
(\mathrm{~m})\end{array}$ \\
\hline "Type I" & 5.6 & 2.18 & 1.1 & 3 \\
"Type II" & 6.75 & 2.15 & 1.7 & 2.84 \\
\hline
\end{tabular}

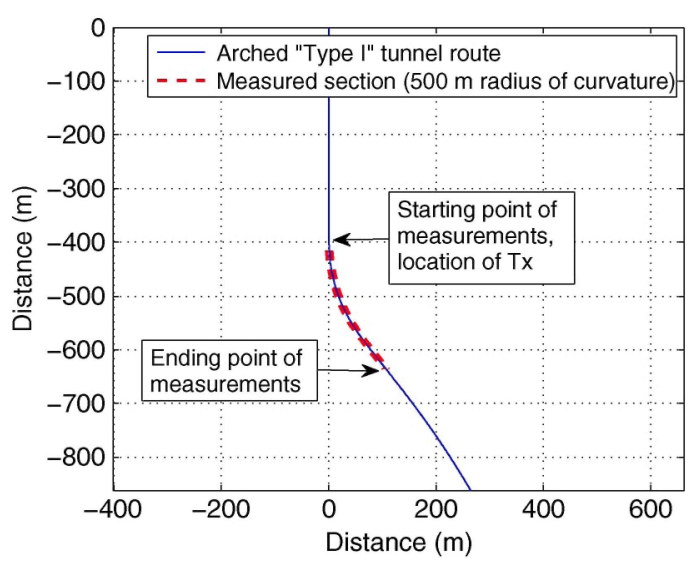

Fig. 4. Top view of the arched "Type I" with a 500-m radius of curvature.

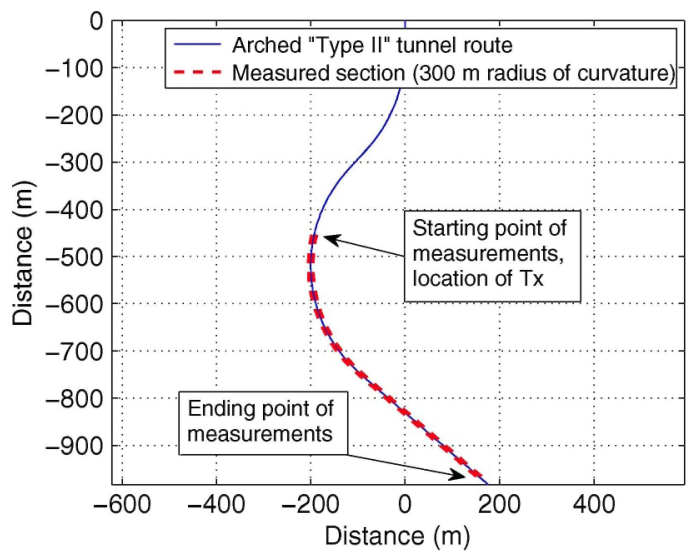

Fig. 5. Top view of the arched "Type II" with a 300-m radius of curvature.

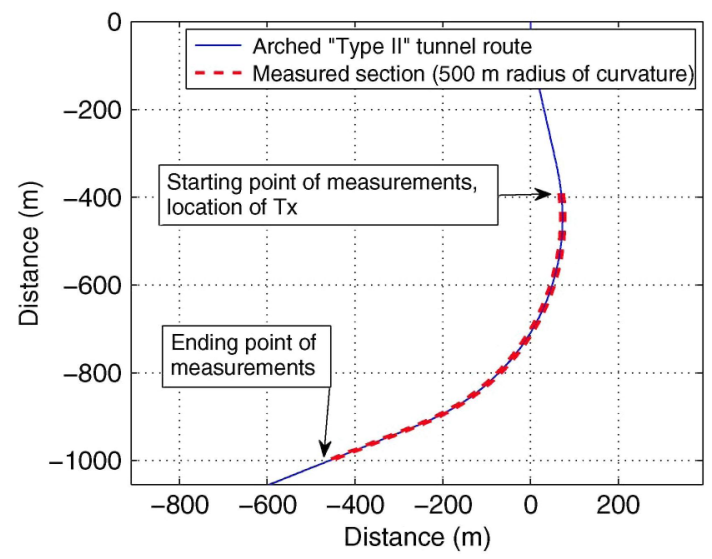

Fig. 6. Top view of the arched "Type II" with a 500-m radius of curvature.

Figs. 4-6 show the top views of the tunnels with different cross sections (arched "Type I" and "Type II") and radii of curvatures (300 and $500 \mathrm{~m}$ ) in this paper, respectively. 


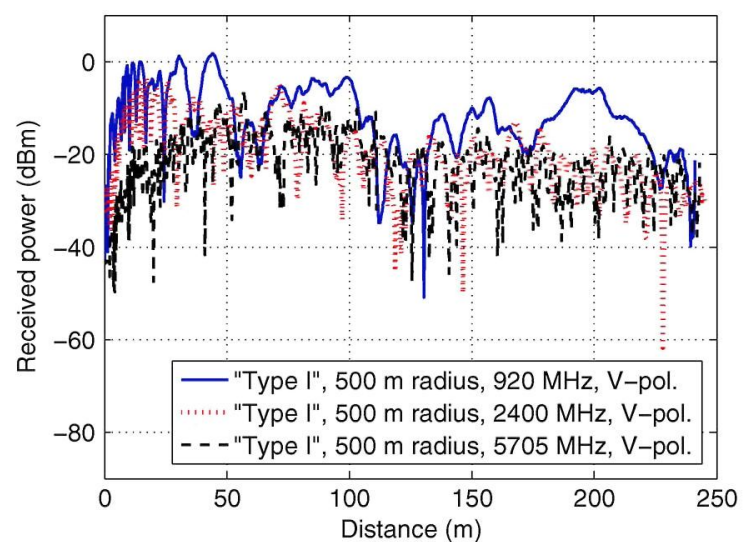

(a)

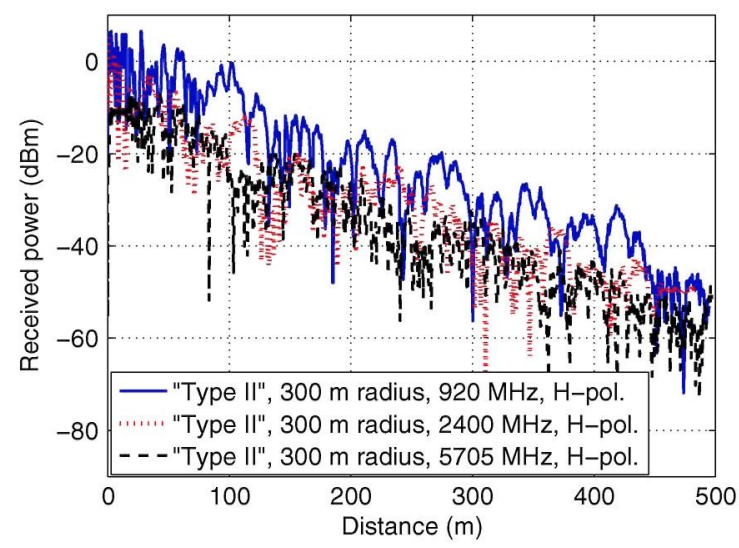

(c)

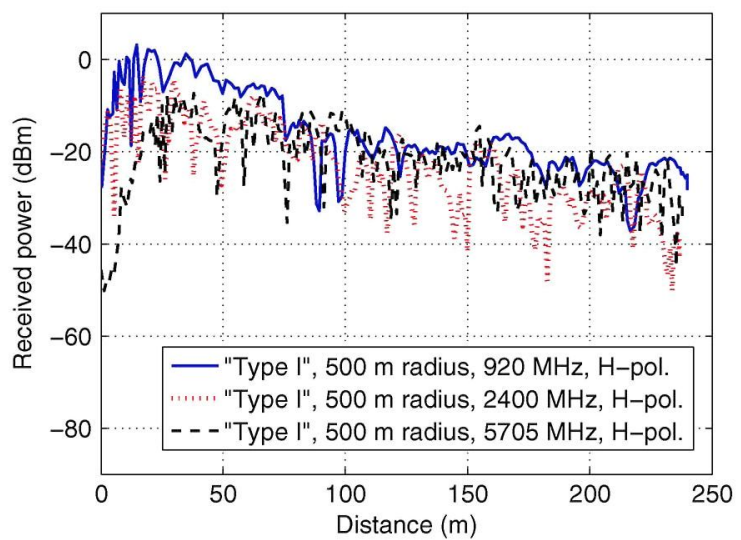

(b)

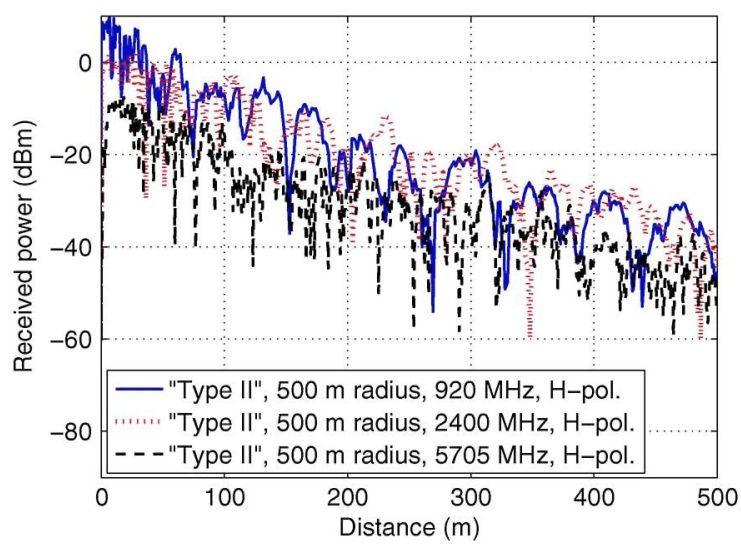

(d)

Fig. 7. Measured received signal power (without small-scale fading) of (a) "Type I" tunnel with a 500-m radius of curvature and V-pol, (b) "Type I" tunnel with a

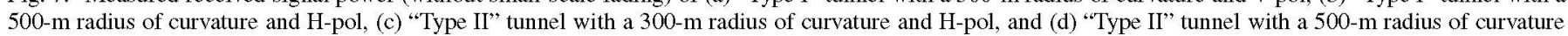
and H-pol.

The measurements are carried out in all the cases shown in Figs. 4-6. In each case, three carrier frequencies-920, 2400, and $5705 \mathrm{MHz}$ - are tested to get the influence of the frequency. In order to observe the impact of the antenna polarization, both vertical polarization (V-pol) and horizontal polarization (H-pol) are measured in the arched "Type I" tunnel. In the arched "Type II" tunnel, the antennas are set to be H-pol. Extensive measurements are made with the train moving very slowly (with the speed around $2 \mathrm{~km} / \mathrm{h}$ ). Therefore, the channel is not time-varying. In order to obtain sufficient samples, the sampling intervals are shorter than one wavelength, and the measurements are made repeatedly. Since this paper focuses on the extra loss resulting from the tunnel curve, small-scale fading is separated from the received power by averaging samples at intervals of 40 wavelengths [42]. The large-scale fading property has been published in our previous work [43], [44]; therefore, it is not included in this paper.

\section{MEAsurement Results}

\section{A. Total Propagation Loss in Curved Tunnels}

The received power (no small-scale fading) in all the measurement campaigns are shown in Fig. 7(a)-(d), respectively. Since the antenna gains are not the same at different frequencies (see Table I), the absolute received power is not comparable. The so-called "propagation loss" in this paper is not an absolute value but an attenuation rate - the slope of the lines (curves) of the received power in Fig. 7-that is not influenced by different antenna gains. By comparing and analyzing the subfigures of Fig. 7, the following interesting observations on the total propagation loss can be summarized. First, The similarity between Fig. 7(a) and (b) indicates that the polarization does not dramatically influence the total propagation loss in the curved arched tunnel. However, since the width of the measured arched "Type I" tunnel is larger than its height, the propagation loss of the V-pol should be larger than that of the H-pol if the tunnel was straight [13]. Thus, the similar total propagation losses in the measured curved tunnel for both polarizations suggest that the extra loss (compared with the case of the straight tunnel) differs with different polarizations, and this point will be studied and justified in Section IV. Second, by comparing Fig. 7(b) with (d), it can be seen that the total attenuation rate in the "Type I" tunnel is lower than that in the "Type II" tunnel. This is mainly because that the arched "Type I" tunnel is closer to a rectangular tunnel. With similar dimensions, the attenuation constant of corresponding mode in the rectangular tunnel is smaller than that in the arched "Type II" tunnel [41]. Third, in each case, the total attenuation rates of different frequencies are similar. However, in straight tunnels, the attenuation rates of different frequencies differ [13]. This means that the extra losses owing to the tunnel curve at different frequencies are not the same. In the latter part of this paper, this observation 


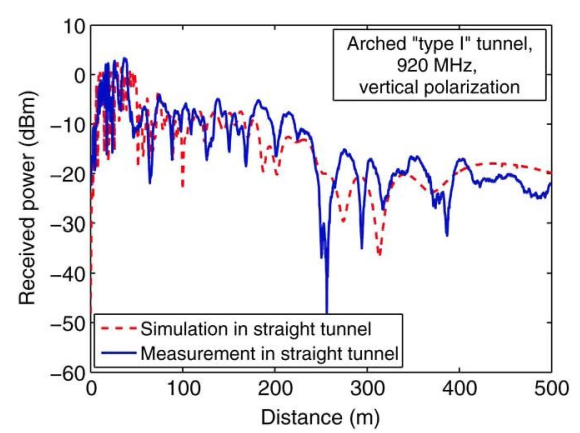

(a)

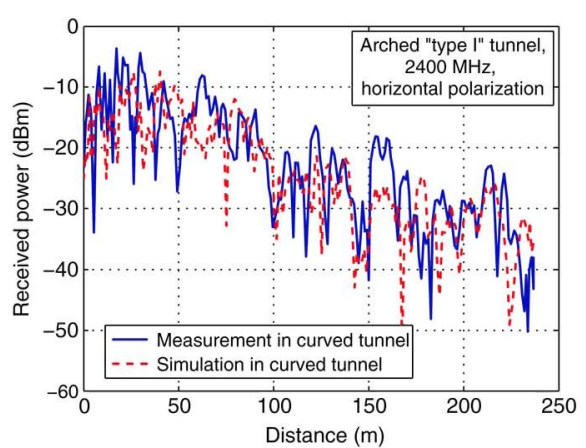

(b)

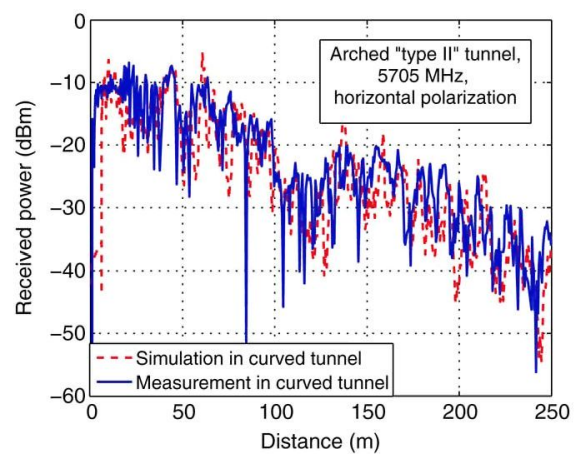

(c)

Fig. 8. Comparisons on the received power (a) between the measurement and the ray-based simulation in the straight arched "type I" tunnel at 920 MHz with V-pol, (b) between the measurement and simulation in the curved arched "type I" tunnel at $2400 \mathrm{MHz}$ with H-pol, and (c) between the measurement and simulation in the curved arched "type II" tunnel at $5705 \mathrm{MHz}$ with H-pol.

is verified by extracting the extra loss in every case, which indeed differs. Fourth, the total propagation losses in this paper with the 300- and 500-m radii of curvatures are larger than the values presented in [32] and [34], in which the radii of curvature are larger than $1 \mathrm{~km}$. This implies that the greater the radius of curvature is, the less the total propagation loss is. Similar conclusions can be found in [35]. For the case shown in Fig. 7(c), the 920- and 5707-MHz waves suffer around 20-dB total losses within a $200-\mathrm{m}$ range, which are similar to the measurement results at $1 \mathrm{GHz}$ and results at $5.8 \mathrm{GHz}$ reported in [36] and [29], respectively. These findings justify the validity of the measurement data and provide a preliminary impression of the extra loss resulting from tunnel curve, which will be analyzed in the following.

\section{B. Extraction of Extra Loss of Tunnel Curve}

In order to extract the extra loss of the tunnel curve from the total received power, a ray-tracing simulator is employed to simulate the reference received signal power by assuming the curved tunnel to be straight. The validity of the ray-tracing simulator in both straight and curved tunnels at different frequencies and polarizations is verified by extensive comparisons with various measurements. Fig. $8(\mathrm{a})-(\mathrm{c})$ are the three instances. Fig. 8(a) shows the comparison on the received power between the measurement and the ray-based simulation in the straight arched "type I" tunnel at $920 \mathrm{MHz}$ with V-pol. The simulator used in this paper is developed in the project TECRAIL [45], based on the image theory, taking into account the antenna patterns, geometry of the tunnel, locations of Tx and Rx, various orders of reflections, and the first order of diffraction. By simulating with three to eight order reflections, we obtain some differences from three to five order reflections but very similar results when using five order or higher order reflections. Since edges are note modeled in the straight tunnel, the diffraction calculation can be eliminated, and considerable computational speed up can be achieved. In the test, simulations with and without diffraction are compared, and the results are very similar. Thus, the ray-based simulations in this paper are with five order reflections and without diffraction, for the sake of the tradeoff between computational load and accuracy. Correspondingly, Fig. 8(b) and (c) shows the comparison in the curved arched

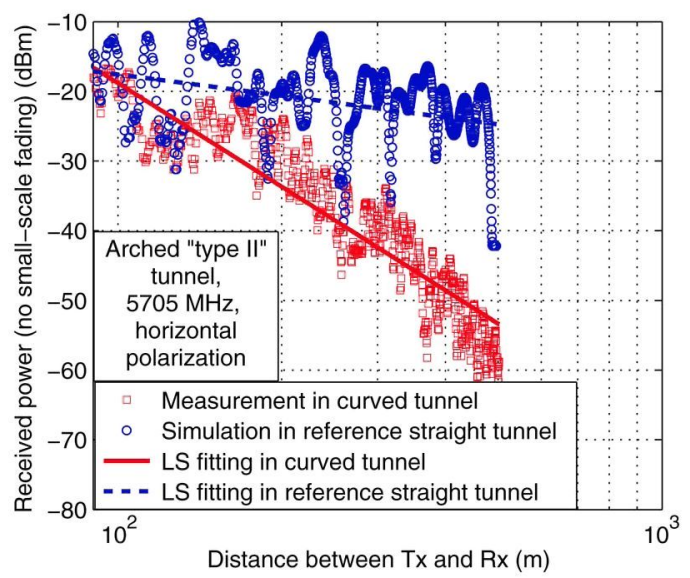

Fig. 9. Comparison on the received power (no small-scale fading) between the measurement in the curved tunnel, simulation in the reference straight tunnel, and corresponding LS fitting lines, respectively, at $5705 \mathrm{MHz}$ with $\mathrm{H}-$ pol in the arched "type II" tunnel.

"type I" tunnel at $2400 \mathrm{MHz}$ with H-pol and the comparison in the curved arched "type II" tunnel at $5705 \mathrm{MHz}$ with H-pol, respectively. Good agreements between the ray-tracing simulations and measurements can be found in the both figures. It is worth noting that the impact of the train is not considered in the simulations with two reasons. First, the simulations and measurements are made with propagation free of obstacles (train) in all cases. Since the train is never in the middle of the propagation path, the impact of it is expected to be limited. Second, the directional receiving antenna is installed on the front of the cabin, which further eliminates the impact of the train. Finally, the good agreements between measurements and simulations without consideration of the train also verify that the impact of the train on the received power in this context is negligible.

With the aid of the ray-tracing simulator, the extra loss of the tunnel curve can be extracted by the following steps. First, use the ray-tracing simulator with the same patterns of antennas used in the measurements to predict the reference received signal power in the equivalent straight tunnel. Second, following the log-distance path-loss model, a line only reflecting the path loss (without shadow fading and smallscale fading) in the reference straight tunnel (assuming the real curved tunnel to be straight in the simulator) can be obtained by 


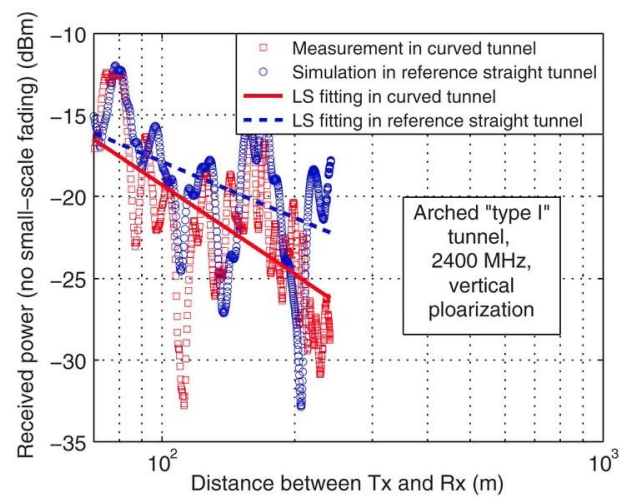

(a)

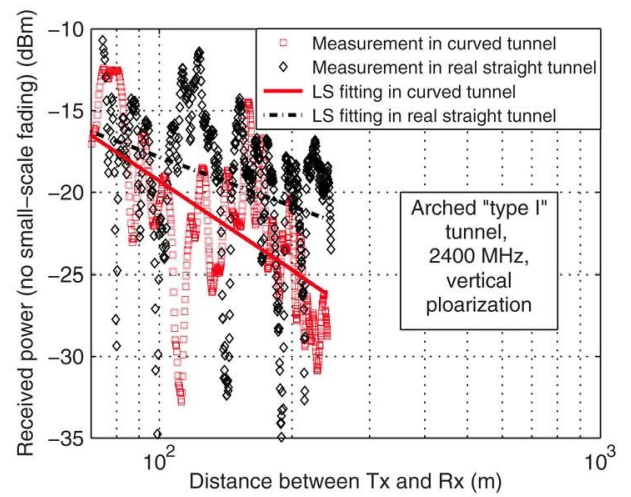

(b)

Fig. 10. Comparison on the received power (no small-scale fading) between the measurement in the curved tunnel, (a) simulation in the reference straight tunnel, (b) measurement in the real straight tunnel, and corresponding LS fitting lines, respectively, at $2400 \mathrm{MHz}$ with V-pol in the arched "type I" tunnel.

using least squares (LS) line fitting for the simulated received power versus the log-distance. Third, the corresponding line can be received by doing the same LS fitting for the measured received power in the real curved tunnel. Fourth, by subtracting the value of the line of the measurement at every distance point (distance between $\mathrm{Tx}$ and $\mathrm{Rx}$ ) from that of simulation at corresponding distance point, the residual virtually represents the extra loss of tunnel curve at every distance point. Fifth, since the coverage range of an antenna inside tunnels is usually several hundreds of meters, the extra loss of tunnel curve is expressed as an attenuation coefficient per $100 \mathrm{~m}$-EL_curve $[\mathrm{dB} / 100 \mathrm{~m}]$. The same or similar terminology is used in [35] and [46], but note that the attenuation unit in $[\mathrm{dB} / 100 \mathrm{~m}]$ or $[\mathrm{dB} / 1000 \mathrm{~m}]$ in these publications are defined by "total loss", including the original loss in the straight tunnel and the extra loss resulting from the curve, whereas the EL_curve $[\mathrm{dB} / 100 \mathrm{~m}]$ defined in this paper means only the "extra loss" of tunnel curve. The advantage of distinguishing the extra loss from the total loss is that the extra loss of tunnel curve can be added to the loss predicted for straight tunnels, so that the existing models for straight tunnels, such as the models in [15]-[25], [27], [28], [40], [41], still can work in curved tunnels.

As an example, Fig. 9 shows the comparison on the received power (no small-scale fading) between the measurement in the curved tunnel, simulation in the reference straight tunnel, and corresponding LS fitting lines, respectively, at $5705 \mathrm{MHz}$ with H-pol in the arched "type II" tunnel. It is worth noting that the ideal solution of extracting the extra loss is to subtract the value of the LS fitting line of the measurement in the curved tunnel from that of the measurement in the same straight tunnel. However, in reality, the same segment of a tunnel cannot be physically straight and curved simultaneously. Thus, the comparison is virtually between the measurement in one straight part and the measurement in another curved part. This may raise problems of comparability due to the space inconsistency between the curved and straight parts. Therefore, the extra loss in this paper is extracted by using the ray-tracing simulator that is validated by extensive measurements because the simulator can build an equivalent straight tunnel of the original curved one, keeping a good space consistency. As an example, Fig. 10(a) and (b) shows the comparisons between

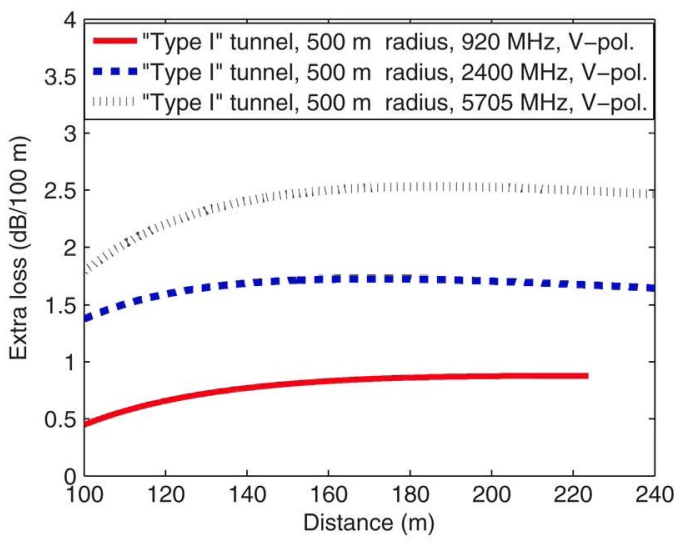

Fig. 11. Extra loss of tunnel curve $[\mathrm{dB} / 100 \mathrm{~m}]$ variation tendency along distance between $\mathrm{Tx}$ and $\mathrm{Rx}$.

the measurements in a curved part and another straight part, as well as the comparisons between the measurements in the curved part and the simulation in the equivalent straight part, respectively, at $2400 \mathrm{MHz}$ with V-pol in the arched "type I" tunnel. The good similarity between Fig. 10(a) and (b) implies that the simulator is adequate to model the real environment; therefore, it can be employed to effectively extract the extra loss.

The values of the extra loss of tunnel curve $[\mathrm{dB} / 100 \mathrm{~m}]$ along the distance between Tx and Rx are shown in Fig. 11. It can be found that the extra loss slightly changes along with the distance. However, the values of the extra loss trend toward a horizontal line, particularly when the distance is longer than 120-140 m. When the distance shorter than 120-140 m, the $\mathrm{Rx}$ is in the near-field zone of propagation inside tunnels [21] and [22], where multiple high-order modes are significant and complicate the propagation. Therefore, the extra loss within this range is not modeled in this paper, and a more detailed analysis is needed for future work. After $120-140 \mathrm{~m}$, the Rx is in the far-field zone where the waveguide propagation is established. Thus, dramatic change of the extra loss in the further distance is not expected because the propagation is dominated by fundamental modes. The trend toward a horizontal line in Fig. 11 verifies this analysis and shows that, in the far-field 
Structure of Measurements and Extra Loss of Tunnel Curve

\begin{tabular}{|c|c|c|c|c|c|c|}
\hline \multirow{2}{*}{\multicolumn{2}{|c|}{$\begin{array}{l}\text { Cross Section of } \\
-\frac{\text { Tunnel }}{\text { Radius of }} \\
\text { Curvature }\end{array}$}} & \multicolumn{2}{|c|}{ Arched "Type I" } & \multicolumn{2}{|c|}{ Arched "Type II" } & ---- \\
\hline & & \multicolumn{2}{|c|}{$500 \mathrm{~m}$} & $300 \mathrm{~m}$ & \multicolumn{2}{|c|}{$500 \mathrm{~m}$} \\
\hline \multicolumn{2}{|c|}{------} & \multicolumn{5}{|c|}{$---\frac{1}{v}-\frac{1}{\nabla}-----\frac{1}{\nabla}---\frac{1}{v}----$} \\
\hline \multicolumn{2}{|c|}{ Polarization } & Horizontal & Vertical & \multicolumn{2}{|c|}{ Horizontal } & \\
\hline \multicolumn{7}{|c|}{------} \\
\hline \multirow{4}{*}{ Frequency } & $\begin{array}{l}920 \\
\mathrm{MHz}\end{array}$ & 1.8 & 0.8 & 5.4 & 3.8 & \multirow{4}{*}{$\begin{array}{c}\text { Extra } \\
\text { Loss } \\
\text { (dB/100m) }\end{array}$} \\
\hline & 2400 & 3.9 & $\frac{-}{1.7}$ & $\frac{--}{5.6}$ & -- & \\
\hline & & & -- & -- & & \\
\hline & $\begin{array}{l}5705 \\
\mathrm{MHz}\end{array}$ & 4.1 & 2.1 & 6.7 & 5.0 & \\
\hline
\end{tabular}

Fig. 12. Structure of measurements and extra loss of the tunnel curve.

TABLE III

Summary of EXtra LoSS OF TUNNEl CURVE IN Literature

\begin{tabular}{|c|c|c|c|c|c|c|c|c|}
\hline \multirow{2}{*}{$\begin{array}{l}\text { Lite- } \\
\text { rature }\end{array}$} & \multirow{2}{*}{$\begin{array}{l}\text { Tunnel } \\
\text { type }\end{array}$} & \multirow{2}{*}{$\begin{array}{l}\text { Cross } \\
\text { section }\end{array}$} & \multirow{2}{*}{$\begin{array}{l}\text { Size }(\mathrm{m}) \\
W \times H\end{array}$} & \multirow{2}{*}{$\begin{array}{l}\text { Radius of } \\
\text { curvature } \\
\text { (m) }\end{array}$} & \multirow[t]{2}{*}{ Pol. } & \multicolumn{3}{|c|}{ Extra loss $(\mathrm{dB} / 100 \mathrm{~m})$} \\
\hline & & & & & & $\begin{array}{c}920 \\
\mathrm{MHz}\end{array}$ & $\begin{array}{l}2400 \\
\mathrm{MHz}\end{array}$ & $\begin{array}{l}5705 \\
\mathrm{MHz}\end{array}$ \\
\hline This one & Subway & Arched "Type I" & $7.59 \times 5.52$ & 500 & $\mathrm{H}$. & 1.8 & 3.9 & 4.1 \\
\hline This one & Subway & Arched "Type I" & $7.59 \times 5.52$ & 500 & V. & 0.8 & 1.7 & 2.1 \\
\hline This one & Subway & Arched "Type II" & $8.41 \times 6.87$ & 300 & H. & 5.4 & 5.6 & 6.7 \\
\hline This one & Subway & Arched "Type II" & $8.41 \times 6.87$ & 500 & $\mathrm{H}$. & 3.8 & 4.4 & 5.0 \\
\hline [35] & Simulation & Rectangular & $8.00 \times 4.00$ & 1000 & H. & 4.5 & 12.2 & - \\
\hline [35] & Simulation & Rectangular & $8.00 \times 4.00$ & 1500 & $\mathrm{H}$. & 3 & 8.1 & - \\
\hline [35] & Simulation & Rectangular & $4.00 \times 4.00$ & 1000 & $\mathrm{H}$. & 2.1 & 6 & - \\
\hline [47] & Simulation & Rectangular & $4.26 \times 2.13$ & 42.6 & H. & 77 & - & - \\
\hline [47] & Simulation & Rectangular & $4.26 \times 2.13$ & 213 & $\mathrm{H}$. & 15 & - & - \\
\hline [47] & Simulation & Rectangular & $4.26 \times 2.13$ & 42.6 & V. & 10 & - & - \\
\hline [48] & Mine & Rectangular & $\begin{array}{l}5-5.7 \times \\
3.5-4.2\end{array}$ & $\begin{array}{c}\text { Around } \\
1000\end{array}$ & $\mathrm{H}$. & - & 14 & - \\
\hline [48] & Mine & Rectangular & $\begin{array}{l}5-5.7 \times \\
3.5-4.2\end{array}$ & $\begin{array}{c}\text { Around } \\
1000\end{array}$ & MV & - & 4.03 & - \\
\hline [48] & Mine & Rectangular & $\begin{array}{l}5-5.7 \times \\
3.5-4.2\end{array}$ & $\begin{array}{c}\text { Around } \\
1000\end{array}$ & $\mathrm{CV}$ & - & 8.35 & - \\
\hline
\end{tabular}

zone, the extra loss is independent from the distance between $\mathrm{Tx}$ and Rx. Moreover, since the propagation between Tx and $\mathrm{Rx}$ is mainly determined by tunnel geometry and material, if the Tx is placed along the curve, the extra loss is not expected to dramatically change when the applied tunnel is of the similar type and curvature of the measured tunnel. Hence, in order to facilitate the use, the median value along the measuring distance is used to represent the typical value of the extra losses $[\mathrm{dB} / 100 \mathrm{~m}]$ in every case. Thus, in the implementation, such as the network planning, the extra loss can be directly added to the propagation loss in the straight tunnel to predict the total propagation loss inside curved tunnels, i.e.,

$$
L[\mathrm{~dB}]=L_{\text {straight }}(d)[\mathrm{dB}]+E L_{\text {curve }}[\mathrm{dB} / 100 \mathrm{~m}] \bullet\left(\frac{d[m]}{100}\right)
$$

where $L$ (in decibels) is the total propagation loss inside curved tunnels, $d$ (in meters) is the distance between Tx and Rx, $L_{\text {straight }}$ (in decibels) is the propagation loss in the straight tunnel, which usually is a function of $d$.

Fig. 12 shows the structure of the whole measurement campaigns and the extracted extra losses of tunnel curve in all the combinations of various cross sections of tunnels, radii of curvature, polarizations, and frequencies. In the measurements, 0.8-6.7 dB/100 m extra losses resulting from the tunnel curve are observed.

A summary of the extra loss of the tunnel curve in literature is given by Table III, where $H$ and $W$ are the height and the width of tunnel, respectively. "H." and "V." are H-pol and V-pol, respectively. "MV" and "CV" are medium height and ceiling height for vertically polarized antennas, respectively. Details can be found in the corresponding literature [35], [47], [48]. Due to different types of tunnel and configurations, the extra loss of tunnel curve from different literature cannot be compared directly; however, the following three common senses still can be found: 1) The extra loss is inversely proportional to the radius of curvature, 2) higher frequency suffers larger extra loss, and 3) H-pol is much more affected by the tunnel curvature than the V-pol. More detailed analysis and interpretations of the correlations between the extra loss and various factors are made in the following.

\section{Analysis of the Extra Loss of Tunnel Curve}

As shown in Fig. 12 and Table III, the extra loss of tunnel curve can be influenced by four factors: radius of curvature, frequency, polarization, and cross section of the tunnel. In the following parts, the correlations between the extra loss and these factors will be quantitatively analyzed based on the extra loss (see Fig. 12) extracted from the measurement results. It is worth noting that, in order to reflect the influence of a certain variable, the comparisons should be limited between the cases that only this variable is different.

\section{A. Extra Loss and Radius of Curvature}

Fig. 13(a) shows the extra loss versus radius of curvature, in the arched "type II" tunnel at various frequencies with $\mathrm{H}$-pol. It can be found that a smaller radius of curvature corresponds to a higher extra loss at all the three frequencies. This observation is in line with common sense. The interesting thing is that the differences of the extra losses between the case of 300-m radius of curvature and the case of 500-m radius of curvature at different frequencies are very similar: $1.6 \mathrm{~dB}$ at $920 \mathrm{MHz}, 1.2 \mathrm{~dB}$ at $2400 \mathrm{MHz}$, and $1.7 \mathrm{~dB}$ at $5705 \mathrm{MHz}$. This indicates that the radius of curvature could influence not only the first-order characters (such as the absolute value of the extra loss), but also the second-order characters of the extra loss (such as the partial derivative of the extra loss for the radius of curvature).

\section{B. Extra Loss and Frequency}

Fig. 13(b) shows the extra loss versus frequency, in the arched "type I" tunnel with a 500-m radius of curvature. Fig. 13(c) shows the extra loss versus frequency in the arched "type II" tunnel with H-pol. From these two figures, it can be noticed that the higher frequency results in a higher extra loss if the other factors do not change. This finding implies that the relatively lower propagation attenuation rate of higher frequencies in the straight tunnels is nullified by the extra loss of tunnel curve. Thus, in the curved tunnel, the total attenuation rates of various frequencies tend to be similar, as shown in Fig. 7. In certain extreme cases, the total attenuation even could increase with the increase in frequency in curved tunnels (e.g., in [46]), which is contradictory to the cases in straight tunnels. Thus, which frequency has the relatively lower attenuation 


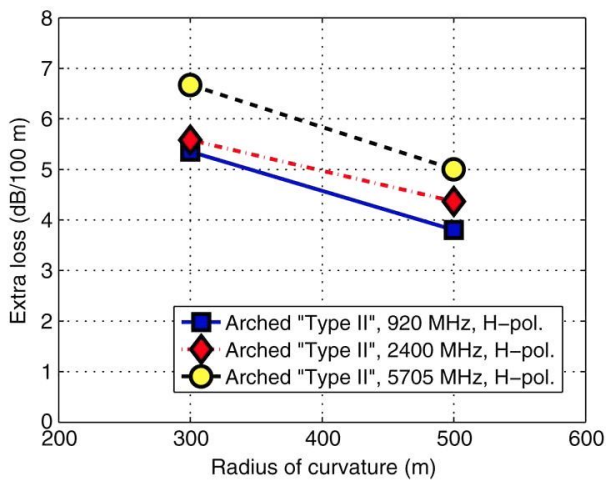

(a)

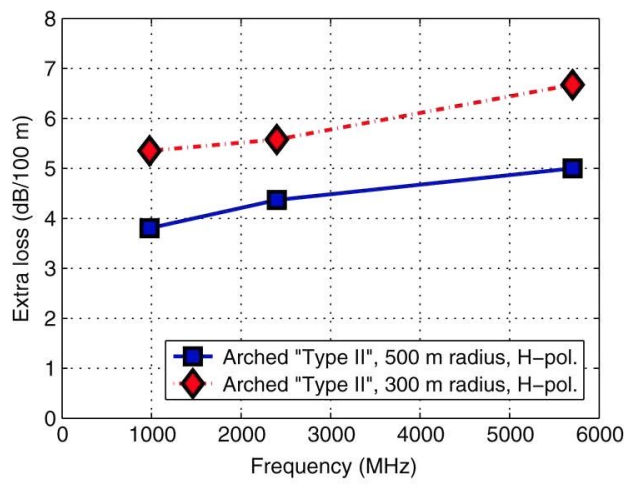

(c)

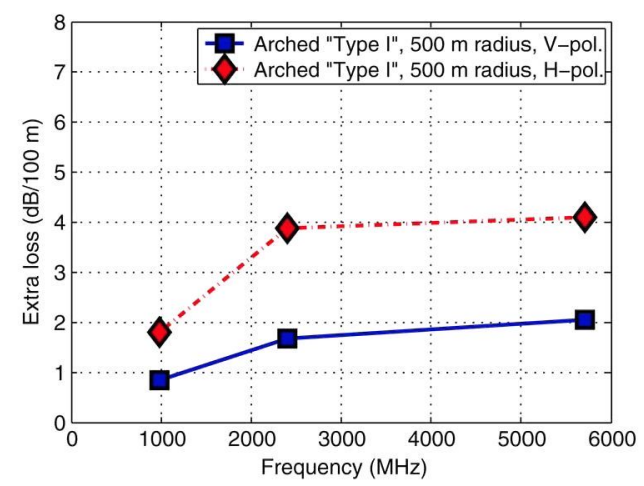

(b)

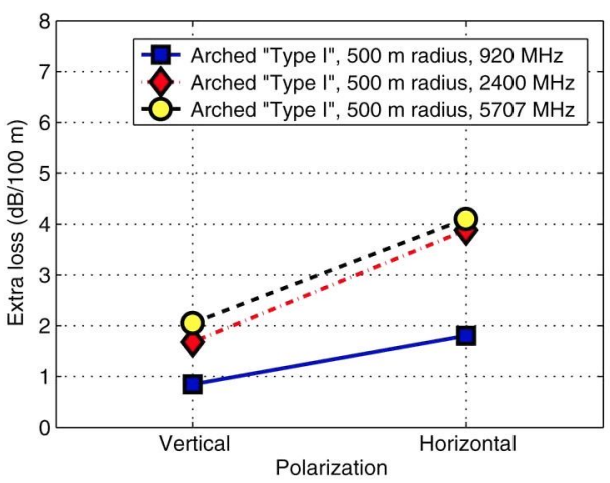

(d)

Fig. 13. (a) Extra loss versus radius of curvature in the arched "type II" tunnel at various frequencies with H-pol. (b) Extra loss versus frequency in the arched "type I" tunnel with a 500-m radius of curvature. (c) Extra loss versus frequency in the arched "type II" tunnel with H-pol. (d) Extra loss versus polarization in the arched "type I" tunnel with a 500-m radius of curvature.

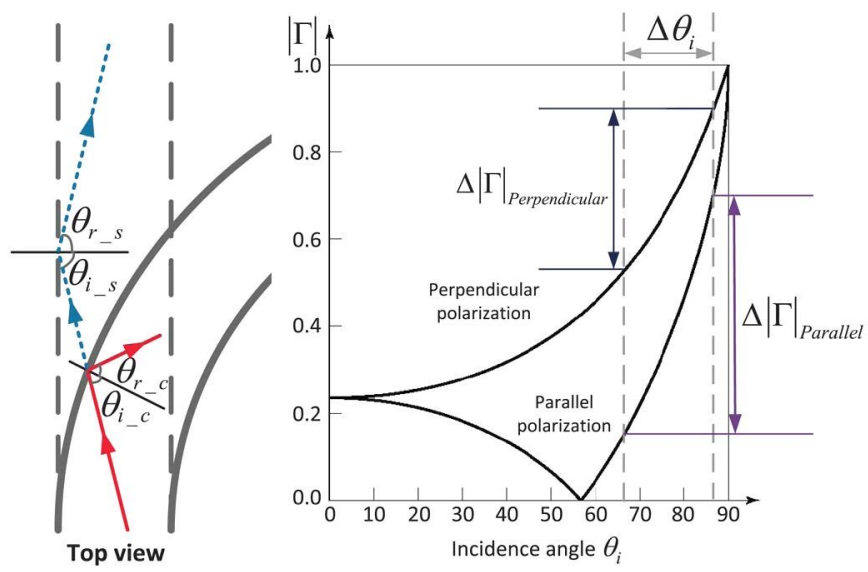

Fig. 14. Top view of a curved tunnel and the referenced straight tunnel Reflection coefficients of perpendicular polarization and parallel polarization.

rate depends on the specific conditions, such as the radius of the curvature, frequency, etc.

\section{Extra Loss and Polarization}

Fig. 13(d) shows the extra loss versus polarization, in the arched "type I" tunnel with a 500-m radius of curvature. It can be observed that the wave propagation has a larger extra loss in the case of H-pol than that in the case of V-pol. This can be interpreted by the change of reflection coefficients. As shown in Fig. 14, the extra loss of the tunnel curve is mainly from the curve in the horizontal direction. In other words, the extra loss mainly comes from the change of the reflection coefficients
$\Delta|\Gamma|$ on the vertical or side tunnel walls with the change of the incidence angles $\Delta \theta_{i}$, i.e.,

$$
\Delta \theta_{i}=\theta_{i \_s}-\theta_{i \_c}=\theta_{r_{-} s}-\theta_{r_{-} c}
$$

where $\theta_{i_{-} c}$ and $\theta_{r_{-} c}$ are the incidence angle and reflection angle on the side walls of a curved tunnel, respectively. Correspondingly, $\theta_{i_{-} s}$ and $\theta_{r_{-} s}$ are the incidence angle and reflection angle on the side walls of the reference straight tunnel. Since the lower order modes (in the modal analysis) are the main modes propagating along the tunnel, $\theta_{i_{-} s}$ of the first-order or secondorder reflections (corresponding to the lower order mode in the modal analysis), is usually not much smaller than $90^{\circ}$. Since the curve in the real tunnels is gradual, $\theta_{i_{c}}$ of the first-order or second-order reflections is usually larger than around $50-60^{\circ}$. Thus, in this interval, the change of the reflection coefficients of the parallel polarization of the side walls (corresponding to $\mathrm{H}$-pol) $\Delta|\Gamma|_{\text {Parallel }}$ is larger than the change of the reflection coefficients of the perpendicular polarization of the side walls (corresponding to $\mathrm{V}$-pol) $\Delta|\Gamma|$ Perpendicular. This leads to the fact that, in the curved tunnel, the wave of $\mathrm{H}$-pol has a larger extra loss than that of V-pol. Hence, V-pol could be a relatively better choice in the curved tunnel because it is not so sensitive to the tunnel curve as the H-pol. Similar observations and interpretations can be found in [31], [47], and [48].

\section{Extra Loss and Cross Section}

Fig. 15 shows the extra loss versus cross section of the tunnel with a 500-m radius of curvature, at various frequencies with $\mathrm{H}$ pol. It can be found that the extra loss in the arched "Type I" 


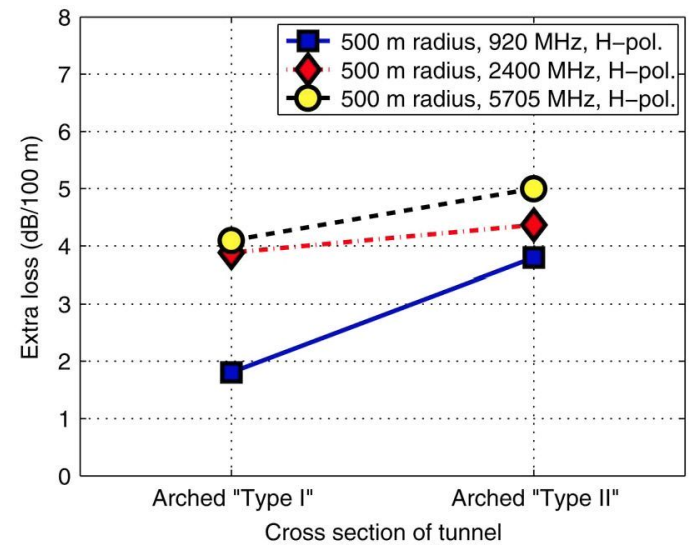

Fig. 15. Extra loss versus cross section of the tunnel with a 500-m radius of curvature at various frequencies with $\mathrm{H}$-pol.

tunnel is smaller than that in the arched "Type II" tunnel at all the three frequencies. This could be linked to the cross sections of tunnels and the polarization. Since the waves in Fig. 15 are with $\mathrm{H}$-pol, the two vertical plane side walls in the arched "Type I" tunnel are more appropriate to establish the horizontally polarized modes than the arched walls in the arched "Type II" tunnel. Similar conclusions can be found in [38] and [49]. This difference could be expected to be slighter in the case that the wave is vertically polarized because the arched roof and plane floor in both types of arched tunnels are similar. Hence, the vertically polarized modes could be established in the similar way. More simulations and measurements are expected to verify this presumption for future work.

\section{DISCUSSION}

Reviewing the measurements and analysis, some final remarks shall be made to provide a better understanding of the results in this paper.

1) This paper presents the first-hand information of the extra loss of tunnel curve based on extensive measurements in two types of arched tunnels. Since the tunnel types, dimensions, frequencies, and measurement campaigns setup are typical and close to the real subway scenarios as well as the communication systems deployed in subway, the values of the extra losses extracted from the measurements (given in Fig. 12) are representative; therefore, they could be involved in the network planning in the corresponding cases directly. If the application environments are dramatically different from the measurement campaigns, the suitability of the values in this paper will decrease. However, these values are still useful to establish statistical models or to validate the theoretical or empirical models on the extra loss of tunnel curve.

2) The measurements are designed and conducted with various combinations of four major conditions: cross section of tunnel, radius of curvature, polarization, and frequency. Nevertheless, not all the combinations are covered due to the limited conditions of the measurements in reality. This is why the measurement campaigns in this paper are described by "comprehensive" and not by "complete." However, these extensive measurements still reflect the correlations between the extra loss and radius of curvature, frequency, polarization, and cross section, respectively. Furthermore, after validation of the ray-tracing simulators using the presented measurement results, it is expected to make more simulations to finally present the panorama of the correlations between extra loss and various factors for future work.

3) In addition to the expression in this paper, there could be other ways to describe the extra loss of tunnel curve. The reasons why we depict the extra loss as an attenuation coefficient per $100 \mathrm{~m}$ are as follows. First, the values of the extra loss $[\mathrm{dB} / 100 \mathrm{~m}]$ seem to be constant, particularly when the distance is longer than $120-140 \mathrm{~m}$ (see Fig. 11), this reasons the way to use the median value along the measuring distance to represent the typical value of the extra loss $[\mathrm{dB} / 100 \mathrm{~m}]$. Second, in the applications, such as the network planning, it is desired to provide an attenuation coefficient versus distance between $\mathrm{Tx}$ and Rx so that the designers can directly add the extra loss of tunnel curve - EL_curve $[\mathrm{dB} / 100 \mathrm{~m}]$ - to the propagation loss in the straight tunnels to get the realize a fast link budge in the curved tunnels, as shown in (1).

4) It may be noticed that the extra loss is not extracted (in this paper) when the distance is shorter than $100-120 \mathrm{~m}$. This is because, in the measuring tunnels at the three frequencies, the region where the distance is shorter than $100-120 \mathrm{~m}$ is the near-field zone, numerical high-order modes exist and dominate. As a consequence, the wave suffers severe loss and complex fading. This makes the extraction of the extra loss of tunnel curve in this area complicated and unreliable. In order to keep the reliability of the results presented in this paper, the modeling of extra loss in the near-field zone is left for future work. With more extensive simulations and measurements, the insight into the propagation mechanisms within this region is expected to establish in the future.

5) To reveal the influence of every single condition, the comparisons in Section IV are limited between the cases that only one condition is different (all the other conditions do not change). This is why the contents in Fig. 13(a)-(d) are reorganized from partial contents in Fig. 12. Not all the extra losses in Fig. 12 are directly compared due to the limited comparability. For instance, between some cases, there are more than one conditions change, and it is hard to distinguish the influence of each condition from the mixed results.

6) The main objective of this paper is the extra propagation loss analysis in curved tunnels. Therefore, additional parameters needed for broadband system analysis are beyond the scope of this paper. However, some of the literature (e.g., in [50] and [51]) points out that the propagation of broadband signals in tunnels is quite good because the delay spread of the signal is very short, as expected in a wave guided propagation medium. Only in large metro stations is there an increase in multipath and delay spread. 


\section{CONCLUSION}

This paper presents the extra loss resulting from the tunnel curve based on extensive measurements in two types of the real subway tunnels at 920,2400, and $5705 \mathrm{MHz}$. Extra losses of $0.8-6.7 \mathrm{~dB} / 100 \mathrm{~m}$ are obtained under various specific conditions. By quantitatively analyzing the correlations between the extra loss and radius of curvature, frequency, polarization, and cross section, respectively, the main results are summarized as follows: A smaller radius of curvature leads to a higher extra loss at all the three frequencies. Moreover, the higher frequency results in a higher extra loss, which means that the relatively lower attenuation rate of higher frequencies in the straight tunnels is reduced by the extra loss of tunnel curve. Unlike in the straight tunnels, here, we cannot conclude which frequency has the relatively lower attenuation rate. In curved tunnels, the attenuation rate depends on the specific conditions, such as the radius of the curvature, frequency, etc. The structures in the horizontal direction, such as the side tunnel walls, influence the extra loss of horizontally polarized wave. Similarly, the structures in the vertical direction, such as the roof and floor, could mainly influence the extra loss of vertically polarized wave. In the curved tunnels, the wave of $\mathrm{H}$-pol has a larger extra loss than that of V-pol. Thus, V-pol could be a relatively better choice in the curved tunnel because it is not so sensitive to the tunnel curve like the H-pol. Given the fact that the real tunnels are always the combinations of curved and straight parts, the best choice would be to use the circular polarization.

Both the quantitative results and qualitative analysis are helpful for statistical modeling and the involvement of the extra loss of tunnel curve in the system design and network planning of communication systems in real subway curved tunnels.

\section{ACKNOWLEDGMENT}

The authors would like to thank Metro de Madrid and, particularly, C. Rodríguez and J. Moreno for their support in performing the propagation measurements, as well as the anonymous reviewers for their thorough reading and constructive comments, which greatly helped to improve this paper.

\section{REFERENCES}

[1] B. Ai, X. Cheng, Z. Zhong, J. Ding, and H. Song, "Social network services for rail traffic applications," IEEE Intell. Syst., vol. 29, no. 6, pp. 63-69, Nov./Dec. 2014.

[2] "Terrestrial Trunked Radio (TETRA); Voice Plus Data (V+D); Designers' Guide; Part 3: Direct Mode Operation (DMO)," 2000.

[3] A. Hrovat, T. Javornik, and G. Kandus, "Adjacent channel interference analyses in tetra direct mode operation," WSEAS Trans. Commun., vol. 7, no. 10 , pp. $1055-1065$, Oct. 2008.

[4] [Online]. Available: http://www.uic.asso.fr

[5] IEEE Standard for Communications-Based Train Control (CBTC) Performance and Functional Requirements, IEEE Std. 1474.1-1999, Dec. 1999.

[6] K. Guan, Z. Zhong, and B. Ai, "Assessment of LTE-R using high speed railway channel model," in Proc. 3 rd Int. Conf. Commun. Mobile Comput., Qingdao, China, 2011, pp. 461-464.

[7] D. J. Smith, Reliability, Maintainability and Risk_Practical Methods for Engineers, 7th ed. Oxford, U.K.: Butterworth-Heinemann, 2005.

[8] B. Ai et al., "Challenges toward wireless communications for high-speed railway," IEEE Trans. Intell. Transp. Syst., vol. 15, no. 5, pp. 2143-2158, Oct. 2014.
[9] Y. Ming, "Robust regional bounding spherical descriptor for 3-D face recognition and emotion analysis," Image Vis. Comput., vol. 35, no. 3, pp. 14-22, Mar. 2015.

[10] Y. Ming and Q. Ruan, "Robust sparse bounding sphere for 3-D face recognition," Image Vis. Comput., vol. 30, no. 8, pp. 524-534, Aug. 2012.

[11] Y. Ming, "Hand fine-motion recognition based on 3D mesh MoSIFT feature descriptor," Neurocomput., vol. 151, no. 2, pp. 574-582, Mar. 2015.

[12] A. E. Forooshani, S. Bashir, D. G. Michelson, and S. Noghanian, "A survey of wireless communications and propagation modeling in underground mines," IEEE Commun. Surveys Tuts., vol. 15, no. 4, pp. 1524-1545, 4th Quart. 2013.

[13] A. Hrovat, G. Kandus, and T. Javornik, "A survey of radio propagation modeling for tunnels," IEEE Commun. Surveys Tuts., vol. 16, no. 2, pp. 658-669, 2nd Quart. 2014.

[14] A. Hrovat, G. Kandus, and T. Javornik, "Path loss analyses in tunnels and underground corridors," Int. J. Commun., vol. 6, no. 3, pp. 136-144, 2012.

[15] C. Briso-Rodriguez, J. Cruz, and J. Alonso, "Measurements and modeling of distributed antenna systems in railway tunnels," IEEE Trans. Veh. Technol., vol. 56, no. 5, pp. 2870-2879, Sep. 2007.

[16] K. Guan, Z. Zhong, J. I. Alonso, and C. Briso, "Measurement of distributed antenna systems at $2.4 \mathrm{GHz}$ in a realistic subway tunnel environment," IEEE Trans. Veh. Technol., vol. 61, no. 2, pp. 834-837, Feb. 2012.

[17] S. H. Chen and S. K. Jeng, "SBR image approach for radio wave propagation in tunnels with and without traffic," IEEE Trans. Veh. Technol., vol. 45 , no. 3 , pp. $570-578$, Aug. 1996

[18] D. Didascalou, T. Schafer, F. Weinmann, and W. Wiesbeck, "Ray-density normalization for ray-optical wave propagation modeling in arbitrarily shaped tunnels," IEEE Trans. Antennas Propag., vol. 48, no. 9, pp. 1316-1325, Sep. 2000.

[19] F. Pallares, F. Juan, and L. Juan-Llacer, "Analysis of path loss and delay spread at $900 \mathrm{MHz}$ and $2.1 \mathrm{GHz}$ while entering tunnels," IEEE Trans. Veh. Technol., vol. 50, no. 3, pp. 767-776, May 2001.

[20] A. V. Popov and N. Y. Zhu, "Modeling radio wave propagation in tunnels with a vectorial parabolic equation," IEEE Trans. Antennas Propag., vol. 48, no. 9, pp. 1403-1412, Sep. 2000.

[21] Y. Zhang and Y. Hwang, "Characterization of UHF radio propagation channels in tunnel environments for microcellular and personal communications," IEEE Trans. Veh. Technol., vol. 47, no. 1, pp. 283-296, Feb. 1998.

[22] Y. Zhang, "Novel model for propagation loss prediction in tunnels," IEEE Trans. Veh. Technol., vol. 52, no. 5, pp. 1308-1314, Sep. 2003.

[23] K. Guan, Z. Zhong, B. Ai, and C. Briso, "Propagation mechanism modelling in the near region of circular tunnels," IET Microw., Antennas Propag., vol. 6, no. 3, pp. 355-360, Feb. 2012.

[24] K. Guan et al., "Complete propagation model in tunnels," IEEE Antennas Wireless Propag. Lett., vol. 12, pp. 741-744, Jul. 2013.

[25] A. Hrovat, G. Kandus, and T. Javornik, "Four-slope channel model for path loss prediction in tunnels at $400 \mathrm{MHz}$," IET Microw., Antennas Propag., vol. 4, no. 5, pp. 571-582, May 2010.

[26] A. Hrovat and T. Javornik, "Analysis of radio propagation models for smart city applications," Int. J. Commun., vol. 7, no. 4, pp. 83-92, 2013.

[27] K. Guan, Z. Zhong, B. Ai, R. S. He, and C. Briso, "Five-zone propagation model for large-size vehicles inside tunnels," Progress Electromagn. Res., vol. 138, pp. 389-405, Jul. 2013.

[28] K. Guan et al., "Complete propagation model structure inside tunnels," Progress Electromagn. Res., vol. 141, pp. 711-726, Aug. 2013.

[29] E. Masson et al., "Radio wave propagation in curved rectangular tunnels at $5.8 \mathrm{GHz}$ for metro applications," in Proc. 11th Int. Conf. ITST, St. Petersburg, Russia, 2011, pp. 81-85.

[30] D. Didascalou, M. Dottling, T. Zwick, and W. Wiesbeck, "A novel rayoptical approach to model wave propagation in curved tunnels," in Proc. IEEE VTS 50 th Veh. Technol. Conf., vol. 4, Amsterdam, The Netherlands, 1999, pp. 2313-2317.

[31] J. S. Lamminmaki and J. J. A. Lempiainen, "Radio propagation characteristics in curved tunnels," Proc. Inst. Elect. Eng.-Microw., Antennas Propag., vol. 145, no. 4, pp. 327-331, Aug. 1998.

[32] M. Lienard, S. Betrencourt, and P. Degauque, "Theoretical and experimental approach of the propagation at $2.5 \mathrm{GHz}$ and $10 \mathrm{GHz}$ in straight and curved tunnels," in Proc. IEEE VTS 50th VTC-Fall, 1999, vol. 4, pp. 2268-2271.

[33] N. Sood, L. Liang, S. V. Hum, and C. D. Sarris, "Ray-tracing based modeling of ultra-wideband pulse propagation in railway tunnels," in Proc. IEEE Int. Symp. APSURSI, Spokane, WA, USA, 2011, pp. 2383-2386. 
[34] W. Te-Shun and Y. Chang-Fa, "Simulations and measurements of wave propagations in curved road tunnels for signals from GSM base stations," IEEE Trans. Antennas Propag., vol. 54, no. 9, pp. 2577-2584, Sep. 2006.

[35] Y. Zhang, Y. Hwang, and P. Ching, "Characterization of UHF radio propagation channel in curved tunnels," in Proc. 7th IEEE Int. Symp. PIMRC, Taipei, 1996, vol. 3, pp. 798-802.

[36] R. Martelly and R. Janaswamy, "Modeling radio transmission loss in curved, branched and rough-walled tunnels with the ADI-PE method," IEEE Trans. Antennas Propag., vol. 58, no. 6, pp. 2037-2045, Jun. 2010.

[37] P. Bernardi, D. Caratelli, R. Cicchetti, V. Schena, and O. Testa, "A numerical scheme for the solution of the vector parabolic equation governing the radio wave propagation in straight and curved rectangular tunnels," IEEE Trans. Antemnas Propag., vol. 57, no. 10, pp. 3249-3257, Oct. 2009.

[38] M. Nilsson, J. Slettenmark, and C. Beckman, "Wave propagation in curved road tunnels," in Proc. IEEE Antennas Propag. Soc. Int. Symp., 1998, vol. 4, pp. 1876-1879.

[39] S. F. Mahmoud, "On modal propagation of high frequency electromagnetic waves in straight and curved tunnels," in Proc. IEEE Antennas Propag. Soc. Int. Symp., 2004, vol. 3, pp. 2963-2966.

[40] K. Guan, Z. Zhong, B. Ai, and C. Briso, "Modeling of the division point of different propagation mechanisms in the near-region within arched tunnels," Wireless Pers. Commun., vol. 68, no. 3, pp. 489-505, Feb. 2013. [Online]. Available: http://dx.doi.org/10.1007/s11277-011-0464-7

[41] K. Guan et al., "Propagation mechanism modeling in the near-region of arbitrary cross-sectional tunnels," Int. J. Antennas Propag., vol. 2012, p. 11, 2012. [Online]. Available: http://dx.doi.org/10.1155/2012/183145

[42] W. C. Y. Lee, "Estimate of local average power of a mobile radio signal," IEEE Trans. Veh. Technol, vol. VT-34, no. 1, pp. 22-27, Feb. 1985.

[43] K. Guan et al., "Measurements and analysis of large-scale fading characteristics in curved subway tunnels at $920 \mathrm{mHz}, 2400 \mathrm{mHz}$, and $5705 \mathrm{mHz}$," IEEE Trans. Intell. Transp. Syst., vol. 16, no. 5, pp. 2393-2405, Oct. 2015.

[44] B. Zhang, Z. Zhong, K. Guan, R. He, and C. Briso, "Shadow fading correlation of multi-frequencies in curved subway tunnels," in Proc. IEEE Conf. ITSC, Qingdao, China, 2014, pp. 1111-1116.

[45] "LTE communication technologies for the automated driving and control railway," [Online]. Available: http://tecrail.lcc.uma.es/

[46] J. Sun and C. Zhang, "Analysis of transmission characteristics of curved tunnel with arbitrary cross section using hybrid mode matching/finite elements approach," in Proc. IEEE Int. Symp. Commun. Inf. Technol., 2005 , vol. 1 , pp. $63-66$.

[47] D. Dudley, M. Lienard, S. Mahmoud, and P. Degauque, "Wireless propagation in tunnels," IEEE Antennas Propag. Mag., vol. 49, no. 2, pp. 11-26, Apr. 2007.

[48] A. Forooshani, R. White, and D. Michelson, "Effect of antenna array properties on multiple-input-multiple-output system performance in an underground mine," IET Microw., Antennas Propag., vol. 7, no. 13, pp. 1035-1044, Oct. 2013.

[49] A. Emslie, R. Lagace, and P. Strong, "Theory of the propagation of UHF radio waves in coal mine tunnels," IEEE Trans. Antennas Propag., vol. AP-23, no. 2, pp. 192-205, Mar. 1975.

[50] R. He et al., "Propagation channel measurements and analysis at $2.4 \mathrm{gHz}$ in subway tunnels," IEEE IET Microw., Antennas Propag., vol. 7, no. 11, pp. 934-941, Aug. 2013.

[51] C. Briso, K. Guan, L. Zhang, and J. Fernandez, "Brodband communications in subway tunnels and stations," in Proc. EUCAP, 2015, pp. 1-5. 